\title{
Hepatic inflammatory pseudotumor mimicking malignancy: the value of differential diagnosis on contrast enhanced ultrasound
}

\author{
Wen-Tao Kong1, Wen-Ping Wang², Hai-Yun Shen¹, Hai-Yan Xue1, Chun-Rui Liu1, Dan-Qing \\ Huang1, Min Wu'
}

${ }^{1}$ Department of Ultrasound, Drumtower Hospital, Medical College of Nanjing University, Nanjing, ${ }^{2}$ Department of Ultrasound, Zhongshan Hospital, Fudan University, Shanghai, China

\begin{abstract}
The aim of this study is to investigate whether the use of contrast enhanced ultrasound (CEUS) can improve the differential diagnostic performance between hepatic inflammatory pseudotumor (HIPT) and other malignant tumors. Material and methods: Forty-four patients with histological proven HIPTs were included in this retrospective study. The features of conventional ultrasound (US) and CEUS were evaluated. Results: Three kinds of enhanced pattern can be seen in the 44 nodules including homogeneous $(n=18,41 \%)$, heterogeneous $(n=16,36 \%)$ and rim-like enhancement $(n=10,23 \%)$. All of the nodules showed hypo-enhancement during the portal and delayed phase. The dominant nodules $(n=29,66 \%)$ presented wash-out within $60 \mathrm{~s}$ after contrast injection. Quick wash-in and wash-out was seen in 18 nodules (41\%). Eighteen nodules (41\%) were correctly diagnosed as HIPT, whereas the remaining 26 cases were misdiagnosed as malignancies $(n=20,45 \%)$ or with an uncertain diagnosis $(n=6,14 \%)$. Conclusion: CEUS was not enough to differentiate HIPT from hepatic malignancies, especially intrahepatic cholangiocarcinoma and liver metastasis. However, some CEUS imaging characteristics may be helpful for HIPT diagnosis.
\end{abstract}

Keywords: contrast enhanced ultrasound; hepatic inflammatory pseudotumor; malignancy; intrahepatic cholangiocarcinoma; liver metastasis

\section{Introduction}

Hepatic inflammatory pseudotumor (HIPT) is a rare benign lesion of liver. It was first reported by Pack and Baker in 1953 [1]. The ratio of male/female in adult HIPT ranged from 1:1 to 3.5:1 [2]. The etiology is still unclear, infection, trauma, immunologic reaction, bile leaking or primary biliary cholangitis being among the incriminated causes [1,3-5]. Clinical manifestation of HIPT may be nonspecific, such as abdominal pain, fever and weight

Received 18.04.2020 Accepted 14.07.2020

Med Ultrason

2021, Vol. 23, No 1, 15-21

Corresponding author: Min-Wu

321, Zhongshan Road, Nanjing,

Jiangsu, China

Phone: +86-025-83304616

E-mail: wuminguyi@163.com loss. Quite a few cases were even asymptomatic and found by ultrasound examination.

The accurate diagnosis of HIPT is difficult, due to the lack of specific laboratory marker and variable imaging findings. There are some nonspecific alterations such as leukocytosis, anemia or an increased in C-reactive protein (CRP) level [6]. On enhanced imaging techniques, it may display variety enhancement patterns, including non-enhancement, hyper-enhancement or peripheral rimlike enhancement [7]. In some cases, HIPT could show quick wash-in during the arterial phase and wash-out during the portal or delayed phase. Therefore, it may be misdiagnosed as a malignant tumor and receive unnecessary surgical resection.

Contrast enhanced ultrasound (CEUS) has been widely used for evaluating microvascularization of tumors. In this research, we summarized the clinical and ultrasound data of 44 patients, with the objective of seeing 
whether CEUS can improve the diagnostic performance of HIPT.

\section{Materials and methods}

\section{Patients}

Forty-four patients ( 30 male, 14 female, mean age $53 \pm 11$ years; age range, 29-73 years) with HIPT were retrospectively analyzed in this study. Inclusion criteria were as follows: 1) having complete clinical and CEUS data; 2) pathologically proven HIPT. The exclusion criteria were: 1) nodules showed no enhancement after contrast administration; 2) typical liver abscesses; 3 ) patients unsuitable for CEUS examination.

The study was approved by the Ethics Committee of Drumtower Hospital and written informed consent was obtained from all patients.

\section{US examination}

Conventional US and CEUS examination were performed by the Philips IU22 unit (Philips Healthcare, Bothell, WA, United States) and the LOGIQ E9 unit (GE Healthcare, Milwaukee, WI, United States) with a 1-5 $\mathrm{MHz}$ convex array probe.

Prior to CEUS, B-mode ultrasonography was first performed to examine the lesion size, location, number, shape, margin and blood flow. A dose of $2.0 \mathrm{ml}$ (for IU22) or $1.2 \mathrm{ml}$ (for LOGIQ E9) of sulphur hexafluoride microbubbles (Sonovue, Bracco, Milan, Italy) was then injected into the cubital vein in bolus via a 20 -gauge needle, followed by a flush of $5 \mathrm{ml}$ of $0.9 \%$ normal saline. Patients were told to breath quietly during the CEUS examination. The static and dynamic images were stored in the hard disk for analysis. In patients with multiple lesions, the largest one was selected as the target for contrast medium.

All of the US and CEUS images were analyzed by two radiologists with at least five years CEUS experience, who were unaware of the clinical and pathologic results of the cases.

\section{Image analysis}

The enhancement phase was analyzed during the arterial phase (10-20 s after injection), portal venous phase $(30-45 \mathrm{~s}$ after injection) and delayed phase ( $>120 \mathrm{~s})$ according to WFSUMB-EFSUMB guidelines [8].

The lesion enhancement patterns were classified as follows: homogeneous enhancement, heterogeneous enhancement and rim-like enhancement. The degree of enhancement was defined in comparison to the adjacent liver parenchyma (hypo-echoic, iso-echoic and hyperechoic). For hyper-enhancement, we further divided into two types. If the nodule enhanced distinctly after contrast injection, it was defined as marked hyper-enhancement.
If the nodule only enhanced a little than the liver parenchyma, then it was defined as mild hyper-enhancement. Quick wash-in and wash-out was defined as showing hyper-enhancement in the arterial phase and became hypoenhanced within $60 \mathrm{~s}$ after the injection of contrast agent. The time to initial enhancement, time to iso-echoic and time to hypo-echoic after contrast agent injection were also recorded for further analysis.

According to the size of the nodule, lesions were divided into three types: $<3 \mathrm{~cm}, 3-5 \mathrm{~cm}$ and $>5 \mathrm{~cm}$. We further compared the conventional US and CEUS characteristics in differential groups.

Hepatic nodules were comprehensive evaluated according to the clinical history, laboratory examination and imaging findings. The following characteristics were considered to be the possible diagnosis of HIPT: 1) no history of hepatitis; 2) normal tumor marker; 3) no signs of malignancy such as echo halo or portal vein tumor thrombosis; 4) mild hyper-enhanced or iso-enhanced during arterial phase; 5) small necrosis area in the nodule. The diagnostic criteria of malignant tumor were as follows: 1) history of hepatitis or extrahepatic malignant tumor; 2) the increase of tumor marker; 3) signs of malignancy on conventional US including echo halo, vascular invasion or bile duct dilatation; 4) quick wash-in and wash-out in CEUS.

\section{Statistical methods}

The data were analyzed by statistical software (SPSS 18.0; SPSS, Chicago, IL). Quantitative data were expressed as means \pm standard deviation (SD). We used independent samples t-test to analyze the quantitative data. Chisquare test and Fisher exact test were used to analyze the categorical data. All tests were two-sided and $\mathrm{p}$ value less than 0.05 were considered statistical significance.

\section{Results}

\section{Patient characteristics}

The characteristics of all patients were summarized in table I. Clinical symptoms include recent history of fever $(n=9)$, jaundice $(n=2)$, and vague upper abdominal discomfort $(\mathrm{n}=6)$. Other 27 cases were asymptomatic and the mass was found in prior ultrasound examination. Five patients had a history of hepatitis B infection. Serum $\alpha$-fetoprotein $(n=1)$ and carbohydrate antigen 19-9 $(n=6)$ levels were slightly elevated in seven patients. Pathological diagnosis of HIPT was obtained by liver resection specimen in 18 cases and percutaneous USguided biopsy in 26 cases. The size of 26 nodules did not change or shrink during the follow-up of more than one year. 
Table I. Baseline characteristics of patients

\begin{tabular}{ll}
\hline Variables & All $(\mathbf{n = 4 4 )}$ \\
\hline Age, years & $53.1 \pm 11.2$ \\
Male: female, n & $30: 14$ \\
History of hepatitis B (yes: no), n & $5: 39$ \\
Liver cirrhosis (yes: no), n & $4: 40$ \\
HBsAg (positive: negative), n & $5: 39$ \\
AFP (positive: negative), n & $1: 43$ \\
Tumor size, cm & $4.0 \pm 2.1$ \\
Number of hepatic tumor size, single: multiple & $29: 15$ \\
Biopsy: hepatectomy & $26: 18$ \\
\hline
\end{tabular}

Data are presented as number or mean \pm SD. HBsAg: Hepatitis B surface antigen; AFP: alpha-fetoprotein, n: number

\section{Conventional US}

Overall, 29 patients had a single nodule, whereas 15 patients had multiple focal nodules. The mean diameter of all nodules was $4.0 \pm 2.1 \mathrm{~cm}$, ranged from 1.0 to $9.2 \mathrm{~cm}$. Thirty-six nodules located in right lobe and 8 nodules located in left lobe.

Conventional gray-scale US showed hyper-echoic in 4 nodules, hypo-echoic in 37 nodules and mixed-echoic in 3 nodules. Thirteen out of 44 nodules were homogeneous and 31 nodules were heterogeneous. Liquefaction necrosis can be seen in four nodules. There were statistically significant differences in shape, margin and echo structure according to the nodule size $(\mathrm{p}<0.05)$. Color Doppler flow imaging (CDFI) showed blood flow signal in 27 nodules, with a mean resistance index (RI) of 0.58 .

\section{CEUS findings}

Table II summarizes the CEUS findings of HIPT. In the arterial phase, the homogeneous (fig 1), heterogeneous (fig 2) and rim-like enhancement (fig 3) were found 18,16 and 10 HIPT, respectively.

Seven $(16 \%)$ of the 44 nodules showed marked hyper-enhanced compared with surrounding liver parenchyma. Sixteen nodules (36\%) were mild hyper-enhanced, 18 nodules (41\%) were iso-enhanced and 3 nodules $(7 \%)$ were hypo-enhanced. However, there were no significant differences of the enhanced pattern $(p=0.721)$ and level between different type groups $(\mathrm{p}=0.061)$. Most nodules $(89 \%, 39 / 44)$ showed an unclear margin during the arterial phase. Necrosis can be seen in 25 out of 44 nodules $(57 \%)$.

During the portal and delayed phase, all of the nodules became hypo-enhanced. The mean time to iso-echoic and hypo-echoic were $28.7 \pm 7.6$ and $42.6 \pm 21.4 \mathrm{~s}$, respectively. Twenty-nine nodules $(66 \%, 29 / 44)$ presented wash-out within $60 \mathrm{~s}$ after contrast injection. Finally, of all the 44 nodules, typical quick wash-in and wash-out pattern was seen in 18 cases $(41 \%, 18 / 44)$.

\section{Diagnostic performance}

Eighteen nodules (41\%) were diagnosed as HIPT based on the clinical history, ultrasound and laboratory examination. However, the diagnosis of malignancy was established in 20 nodules (46\%), including hepatocellular carcinoma (HCC) $(5 \%, 2 / 44)$, intrahepatic cholangiocarcinoma (ICC) $(27 \%, 12 / 44)$, and liver metastasis $(14 \%$, $6 / 44)$. The rest of 6 cases $(13 \%)$ were still indeterminate after CEUS administration.

\section{Pathological findings}

The pathological findings of HIPTs in this group were as follows: 1) nodules contained a mixture of inflammatory cells with a predominance of mature plasma cells; 2) granulomatous inflammation; 3) solidified necrosis; 4) proliferation of fibrous tissue.

\section{Discussion}

HIPT is a rare, benign, non-neoplastic mass. No uniform criteria have been recommended for diagnosis and classification. HIPT has a wide variability in histologic features. Balabaud et al summarized 5 histologic subgroups of HIPT [9]: a plasma cell-rich subgroup with aggregates of or diffuse lymphocytes, a mixed inflammatory cell subgroup, a granulomatous subgroup, a granulomatous subgroup with eosinophils and a predominantly purulent subgroup. Therefore, it may show different imaging signs at different stage of the disease. According to the previous studies $[7,10]$, HIPT revealed variable appearances on image findings. It depends on the proportion and distribution of inflammatory cells, vessels, fibrosis and necrosis within the lesion in the course of disease progression. For example, one type of HIPT is the solitary necrotic nodule, which is easier to be diagnosed on enhanced imaging. No enhancement can be seen in the nodule after the contrast injection because of the completely coagulative necrosis.

In view of the fact that some cases may regress spontaneously or after conservative treatment [4], surgical excision is not the preferred treatment option. However, in most cases, the preoperative diagnosis of HIPT is difficult. The radiological features of HIPT are usually nonspecific [6]. It often shows hypodensity or iso-density on a no contrast computed tomography (CT) scan. On magnetic resonance imaging (MRI), HIPT may show hypointense on T1-weighted images and hyperintense on T2weighted images. Usually, HIPTs may mimic malignant tumors, thus resulting in unnecessary invasive treatment.

Many cases of HIPT were first detected by ultrasonography, but the imaging features were non-specific. HIPT may show hyper-echoic, hypo-echoic, or mix-echoic findings with an obscure margin on gray-scale US. The 
Hepatic inflammatory pseudotumor mimicking malignancy: differential diagnosis, CEUS

Table II. Conventional ultrasound and CEUS characteristics of nodules

\begin{tabular}{|c|c|c|c|c|c|}
\hline Characteristics & Overall & $<3 \mathrm{~cm}$ & $3-5 \mathrm{~cm}$ & $>5 \mathrm{~cm}$ & $\mathbf{p}$ \\
\hline \multicolumn{6}{|l|}{ Echo intensity } \\
\hline High echogenic & 4 & 2 & 1 & 1 & \multirow[t]{3}{*}{0.409} \\
\hline Low echogenic & 37 & 14 & 11 & 12 & \\
\hline Mixed echogenic & 3 & 0 & 1 & 2 & \\
\hline \multicolumn{6}{|l|}{ Echo structure } \\
\hline Homogeneous & 13 & 9 & 4 & 0 & \multirow[t]{2}{*}{0.003} \\
\hline Heterogeneous & 31 & 7 & 9 & 15 & \\
\hline \multicolumn{6}{|l|}{ Shape } \\
\hline Regular & 19 & 11 & 6 & 2 & \multirow[t]{2}{*}{0.009} \\
\hline Irregular & 25 & 5 & 7 & 13 & \\
\hline \multicolumn{6}{|l|}{ Margin } \\
\hline Clear & 15 & 8 & 6 & 1 & \multirow[t]{2}{*}{0.024} \\
\hline Unclear & 29 & 8 & 7 & 14 & \\
\hline \multicolumn{6}{|l|}{ Enhanced pattern in arterial phase } \\
\hline Homogeneous enhancement & 18 & 8 & 5 & 5 & \multirow[t]{3}{*}{0.721} \\
\hline Heterogeneous enhancement & 16 & 2 & 5 & 9 & \\
\hline Rim-like enhancement & 10 & 7 & 3 & 0 & \\
\hline \multicolumn{6}{|l|}{ Enhanced level in arterial phase } \\
\hline Hypoechoic & 3 & 0 & 0 & 3 & \multirow[t]{4}{*}{0.061} \\
\hline Marked hyperechoic & 7 & 4 & 0 & 3 & \\
\hline Mild hyperechoic & 16 & 7 & 5 & 4 & \\
\hline Isoechoic & 18 & 5 & 8 & 5 & \\
\hline \multicolumn{6}{|l|}{ Margin after enhancement } \\
\hline Clear & 5 & 2 & 2 & 1 & \multirow[t]{2}{*}{0.761} \\
\hline Unclear & 39 & 14 & 11 & 14 & \\
\hline \multicolumn{6}{|l|}{ Echo in portal and delayed phase } \\
\hline Hypoechoic & 37 & 15 & 9 & 13 & - \\
\hline \multicolumn{6}{|l|}{ Necrosis in tumor } \\
\hline Yes & 25 & 9 & 9 & 7 & \multirow[t]{2}{*}{0.493} \\
\hline No & 19 & 7 & 4 & 8 & \\
\hline Time to isoechoic & $28.7 \pm 7.6$ & $28.2 \pm 6.4$ & $26.9 \pm 5.4$ & $31.4 \pm 10.5$ & 0.374 \\
\hline Time to hypoechoic & $42.6 \pm 21.4$ & $42.5 \pm 15.6$ & $33.7 \pm 20.1$ & $50.2 \pm 26.8$ & 0.201 \\
\hline \multicolumn{6}{|l|}{ Quick wash-in and wash-out } \\
\hline Yes & 18 & 10 & 3 & 5 & \multirow[t]{2}{*}{0.081} \\
\hline No & 26 & 6 & 10 & 10 & \\
\hline \multicolumn{6}{|l|}{ Quick wash-out } \\
\hline Yes & 29 & 13 & 8 & 8 & \multirow[t]{2}{*}{0.249} \\
\hline No & 15 & 3 & 5 & 7 & \\
\hline \multicolumn{6}{|l|}{ Diagnosis } \\
\hline HIPT & 18 & 6 & 7 & 5 & \multirow[t]{3}{*}{0.456} \\
\hline MT & 20 & 8 & 5 & 7 & \\
\hline Uncertain & 6 & 2 & 1 & 3 & \\
\hline
\end{tabular}

HIPT: hepatic inflammatory pseudotumor; MT: malignant tumor

inside of the nodules was usually heterogeneous. Some nodules can show internal blood flow on CDFI, with low RI. Our study found that larger nodules were prone to have an irregular shape, unclear margin and heterogeneous echo.

The US contrast agent Sonovue has been widely used in the diagnosis of focal liver lesions. In this study, we summarized the data of 44 cases and wanted to investigate whether CEUS could help to improve the diagnos- tic performance of HIPT. Unlike other authors [10], our study demonstrated that homogeneous and heterogeneous enhancement was the most common enhanced pattern. Nodule size has little effect on the enhancement pattern and level. The reason of enhancement during the arterial phase is due to the inflammatory blood vessels. It is worth noting that although most of the cases showed enhancement after contrast injection, the enhancement level was not very high. Thirty-four nodules $(77 \%)$ were 


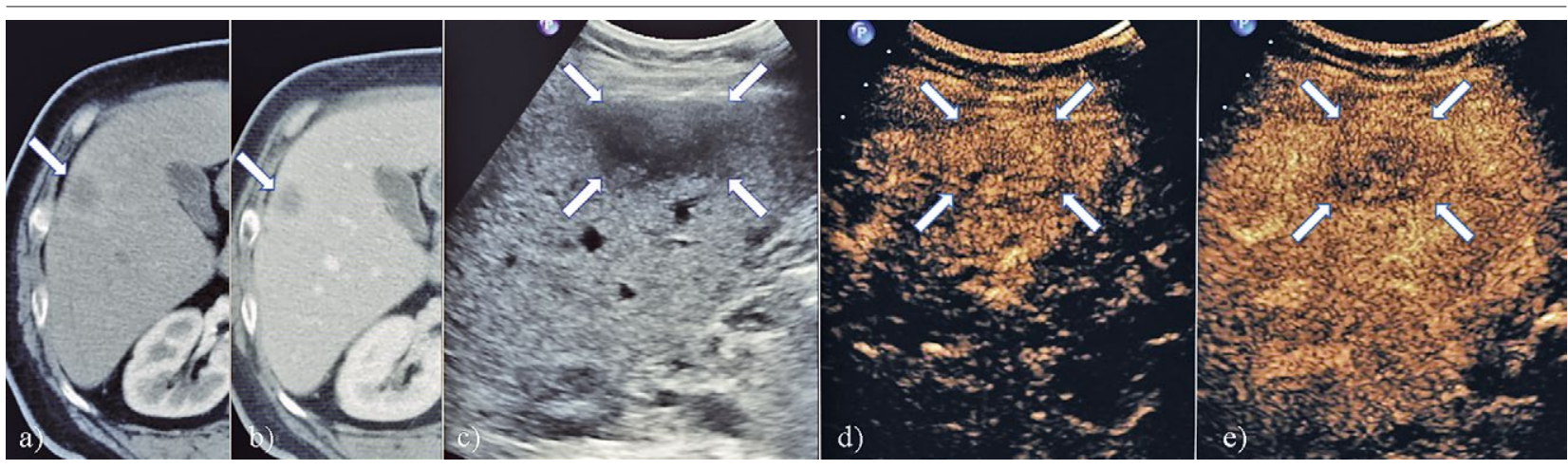

Fig 1. Abdominal computed tomography (CT) showed a mass with the size of $2.5 \mathrm{~cm}$ in segment VIII of the liver. The nodule showed a rim-like enhancement in the arterial phase (a) and washout in the portal phase (b). On B-mode ultrasound (US), the nodule revealed low echo with unclear margin (c). Contrast-enhanced ultrasound (CEUS) revealed a homogeneous hyper-enhancement (d) at 25 seconds after injection of the contrast medium followed by wash-out in the later arterial phase at 30 seconds (e).
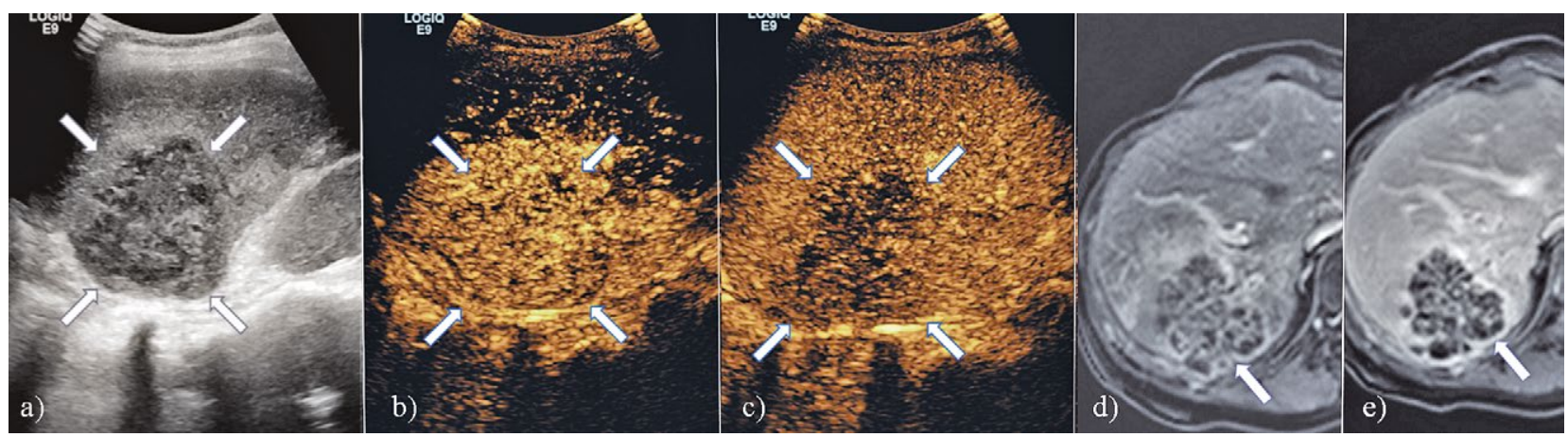

Fig 2. B-mode US showed an inhomogeneous hypo-echoic lesion in the segment VII (a). CEUS showed the nodule enhanced heterogeneously with a small necrotic area in it (b). The lesion washed out quickly, and at 55 seconds it was hypo-echoic with respect to the surrounding liver parenchyma (c). However, on contrast-enhanced magnetic resonance (MR), the nodule showed a honeycomb-like enhancement in the arterial phase (d) and hypo-enhancement in the portal phase (e).

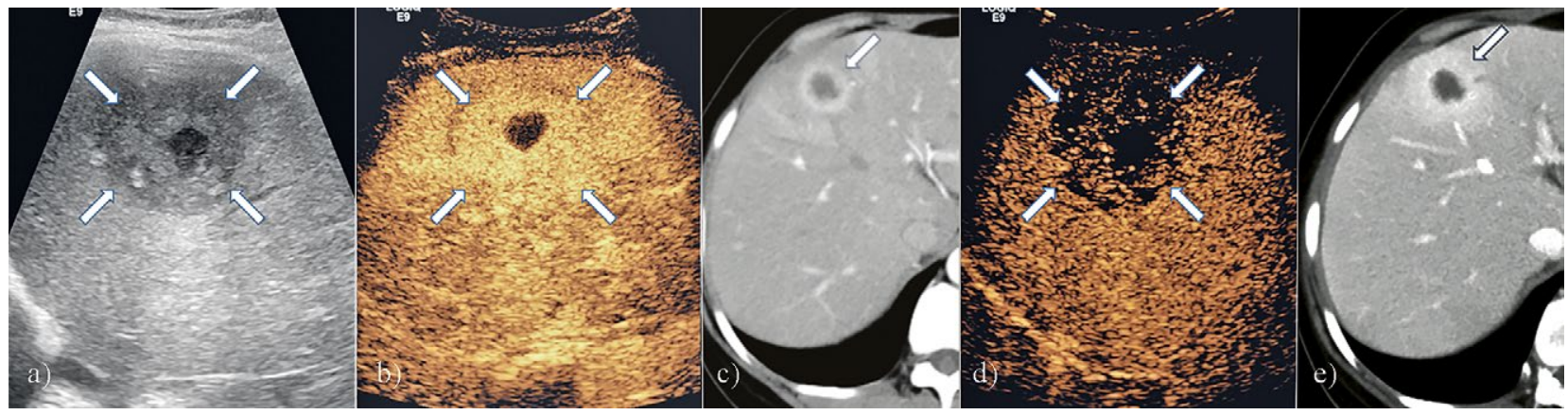

Fig 3. B-mode US image revealed a mixed echo nodule with a thick wall in the right liver (a). Both CEUS and enhanced CT showed rim-like enhancement in the arterial phase $(b, c)$ and washout in the portal phase $(d, e)$.

mild hyper- or iso- enhanced. This phenomenon may reflect the relatively poor blood supply of HIPT, indicating the density of inflammatory blood vessels is not as rich as tumor vascularity. On the other hand, all of the 44 cases showed hypo-enhancement during portal and delayed phase. Rapid wash-out of enhancement may be related to the arterial vascular volume of the tumor, the availability of draining veins, and the characteristics of the microvasculature within the tumor [11]. The cause of quick wash-out in HIPT is not very clear. Relatively poor arterial vascular volume may be one of the reasons. According to the characteristics of hypo-vascular nodules, it may be explained why HIPT is easily misdiagnosed as ICC or liver metastasis. Zhang et al [12] also pointed 
out the centrifugal wash-out was a useful clue in differentiating HIPT from HCC. However, we did not find this characteristic in our study.

More than one paper has mentioned the rapid wash-in and wash-out as the main CEUS characteristic of HIPT $[7,12]$. However, most malignancies show this feature, except a few cases such as highly differentiated HCC $[8,13]$. This imaging feature may be the reason why quite a few cases were misdiagnosed as malignancies. In our study, most common initial misdiagnosis based on conventional US and CEUS findings was ICC (27\%), followed by liver metastasis (14\%). Findings on enhanced CT or MRI of HIPT may also mimic malignant liver tumors due to the same reason. However, Guo et al pointed that inflammatory lesions rather than malignant lesions showed the features of hypoenhancement in arterial phase and portal venous phase and isoenhancement in the delayed phase $(\mathrm{p}<0.05)$ [14].

Another characteristic of HIPT was the internal nonenhanced area, which may be correlated with focal coagulation necrosis or the abscess formation [3]. This phenomenon can be seen in about $57 \%$ nodules in our study. Unlike HCC, the necrosis of HIPT was scattered in the tumor, not necessarily in the center. There was not much liquefaction necrosis, which cannot be easily detected by conventional US.

Due to the fact that obtaining a preoperative diagnosis of HIPT is difficult, the percutaneous liver biopsy is important. The value of needle biopsy for HIPT has some disadvantages and disputes, such as the sampling error, biopsy complication, or tumor seeding when suspicious of malignancy. Some reports evidenced the rate of the correct diagnosis of HIPT by needle biopsy is not very high [15]. In our study, 26 cases obtained a correct pathological diagnosis by needle biopsy, without any complications. We consider biopsy to be a better method for the diagnosis of IPT, thereby avoiding unnecessary surgery.

We should acknowledge there were some limitations in our study. First, it was a retrospective study involving a relatively small number of cases within 12 years. Although we have the clinical and CEUS data of the total 44 patients, other imaging data such as CT or MRI were not complete. Therefore, we did not compare the diagnostic performance of CEUS with other techniques. Second, for further analysis, we divided the hyper-enhanced pattern into two groups including marked and mild enhancement. The intensity of contrast uptake was estimated by observation. Because of the usage of a different ultrasonic machine and contrast dose, we did not use software-based quantitative analysis to quantify the enhanced intensity. The grouping may be not objective.
In conclusion, CEUS is not enough to differentiate HIPT from other hepatic malignancies, especially ICC and liver metastasis. However, some CEUS imaging characteristics maybe clues for the diagnosis of HIPT whenever the following are present: mild hyper-enhanced or iso-enhanced, quick wash-out, unclear border after contrast administration, and small internal non-enhanced area in the nodule. Meanwhile, if there is no hepatitis history and the tumor markers are normal, the diagnosis of HIPT should be under consideration. Further ultrasoundguided percutaneous liver biopsy is necessary to avoid an unnecessary hepatectomy.

Acknowledgements: This study was funded by the National Natural Science Foundation of China (81671701) and the Nanjing Medical Science and technique Development Foundation (QXR17011)

\section{Conflict of interest: None}

\section{Reference}

1. Pack GT, Baker HW. Total right hepatic lobectomy; report of a case. Ann Surg1953;138: 253-258.

2. Shek TW, Ng IO, Chan KW. Inflammatory pseudotumor of the liver. Report of four cases and review of the literature. Am J Surg Pathol 1993;17:231-238.

3. Chang SD, Scali EP, Abrahams Z, Tha S, Yoshida EM. Inflammatory pseudotumor of the liver: a rare case of recurrence following surgical resection. J Radiol Case Rep 2014;8:23-30.

4. Motojuku M, Oida Y, Morikawa G, et al. Inflammatory pseudotumor of the liver: case report and review of literature. Tokai J Exp Clin Med 2008;33:70-74.

5. Zhao J, Olino K, Low LE, Qiu S, Stevenson HL. Hepatic inflammatory pseudotumor: an important differential diagnosis in patients with a history of previous biliary procedures. ACG Case Rep J 2019;13:e00015.

6. Yin L, Zhu B , Lu XY, Lau WY, Zhang YJ. Misdiagnosing hepatic inflammatory pseudotumor as hepatocellular carcinoma: A case report. JGH Open 2017;1:76-78.

7. Kong WT, Wang WP, Cai H, Huang BJ, Ding H, Mao F. The analysis of enhancement pattern of hepatic inflammatory pseudotumor on contrast-enhanced ultrasound. Abdom Imaging 2014;39:168-174.

8. Claudon M, Dietrich CF, Choi BI, et al; World Federation for Ultrasound in Medicine; European Federation of Societies for Ultrasound. Guidelines and Good Clinical Practice Recommendations for Contrast Enhanced Ultrasound (CEUS) in the Liver - Update 2012: A WFUMBEFSUMB Initiative in Cooperation with Representatives of AFSUMB, AIUM, ASUM, FLAUS and ICUS. Ultrasound Med Biol 2013;39:187-210.

9. Balabaud C, Bioulac-Sage P, Goodman ZD, Makhlouf HR. Inflammatory pseudotumor of the liver: a rare but dis- 
tinct tumor-like lesion. Gastroenterol Hepatol (NY) 2012;8:633-634.

10. Park JY, Choi MS, Lim YS, et al. Clinical features, image findings, and prognosis of inflammatory pseudotumor of the liver: a multicenter experience of 45 cases. Gut Liver 2014;8:58-63.

11. Murphy-Lavallee J, Jang HJ, Kim TK, Burns PN, Wilson SR. Are metastases really hypovascular in the arterial phase? The perspective based on contrast-enhanced ultrasonography. J Ultrasound Med 2007;26:1545-1556.

12. Zhang X, Tang S, Huang L, Yu H. Contrast-Enhanced Sonographic Characteristics of Hepatic Inflamma- tory Pseudotumors. J Ultrasound Med 2016;35:20392047.

13. Feng Y, Qin XC, Luo Y, Li YZ, Zhou X. Efficacy of Contrast-Enhanced Ultrasound Washout Rate in Predicting Hepatocellular Carcinoma Differentiation. Ultrasound Med Biol 2015;41:1553-1560.

14. Guo Y, Qin X, Chen S, Liu X, Gu P. Diagnosis efficacy of CEUS for hepatic inflammatory lesions. J Clin Lab Anal 2020;34:e23231.

15. Hosler GA, Steinberg DM, Sheth S, Hamper UM, Erozan YS, Ali SZ. Inflammatory Pseudotumor: A diagnostic dilemma in cytopathology. Diagn Cytopathol 2004;31:267-270. 